\title{
Acknowledgement to Reviewers of Languages in 2020
}

\author{
Languages Editorial Office
}

Citation: Languages Editorial Office. 2021. Acknowledgement to

Reviewers of Languages in 2020.

Languages 6: 21. https://doi.org/

10.3390/languages6010021

Published: 29 January 2021

Publisher's Note: MDPI stays neutral with regard to jurisdictional claims in published maps and institutional affiliations.

Copyright: (C) 2021 by the author. Licensee MDPI, Basel, Switzerland. This article is an open access article distributed under the terms and conditions of the Creative Commons Attribution (CC BY) license (http://creativecommons.org/licenses /by/4.0/).

MDPI AG, St. Alban-Anlage 66, 4052 Basel, Switzerland

The editorial team greatly appreciates the reviewers who have dedicated a considerable amount of time and expertise to the journal's rigorous editorial process over the past 12 months, regardless of whether the papers have been finally published or not. In 2020, a total of 75 papers were published in the journal, with an average time to first decision of 26 days and an average time of 69 days from submission to publication. The editors would like to express their sincere gratitude to the following reviewers for their generous contribution to Languages in 2020:

Aalberse, Suzanne

Aguado-Orea, Javier

Ahn, Hyunah

Alexiadou, Artemis

Amenós-Pons, José

Anastasio, Simona

Anderson, Wendy

Andrews, Edna

Andrews, Jean

Bai, Jianhua

Barkanyi, Zsuzsanna

Barra, Mario

Bellamy, Kate

Biberauer, Theresa

Blasi, Damián

Blithe, Sarah

Bobkina, Jelena

Bonaventura, Patrizia

Bongiovanni, Silvina

Boomershine, Amanda

Brandl, Anel

Broś, Karolina

Buac, Milijana

Bürki, Yvette

Cabal-Jiménez, Munia

Camacho, Jose

Carvalho, Ana Maria

Celata, Chiara

Chamorro, Mónica

Charalambidou, Anna

CHIN, Chi On

Christensen, Ken Ramshøj

Christiner, Markus

Costa, Rute

\author{
Cristobal, Laura Rodrigo \\ Crookes, Graham \\ Darcy, Isabelle \\ De Leeuw, Esther \\ DeCastro-Arrazola, Varun \\ Delgado, Rodrigo \\ Dubiel, Bozena \\ Dumont, Jennifer \\ Ebert, Shane \\ Eddington, David Ellingson \\ Edmonds, Amanda \\ Elliott, A. Raymond \\ Erbaugh, Mary S. \\ Fabra, Lucrecia Rallo \\ Face, Timothy \\ Fagyal, Zsuzsanna \\ Flores, Cristina \\ Freeman, Max \\ Fricke, Melinda \\ Frolova, Anna \\ Fuchs, Zuzanna \\ Fuertes-Olivera, Pedro A. \\ Galatanu, Olga \\ García, Jesús Manuel Nieto \\ García-del-Real, Isabel \\ Geeslin, Kimberly L. \\ Golcher, Felix \\ Green, David \\ Guillaume, Benedicte \\ Guynes, Kristen \\ Heil, Jeanne \\ Henry, Nicholas \\ Hobson, Hannah \\ Holliday, Jeffrey
}


Hong, Ng Chiew

Horvathova, Bozena

Ivanova, Olga

Jean-Rémi, Lapaire

Jiang, Wendy

Jin, Li

Kanwit, Matthew

Kissling, Elizabeth

Kopečková, Romana

Koronkiewicz, Bryan

Lea, Brooke

Leal, Tania

Lee, So

Leivada, Evelina

Li, Shuai

Limerick, Philip P

Lipski, John

Llama, Raquel

Llanos, Fernando

Llompart, Miquel

Lochtman, Katja

Long, Avizia

Lord, Gillian

Luo, Han

MacSwan, Jeff

Martínez-León, Natalia

Matthews, Stephen

Mayer, Elisabeth

Mayr, Robert

Mazzaro, Natalia

Monteagudo, Henrique

Morris, Richard

Mougeon, Francoise P

Mudambi, Aradhana

Munarriz, Amaia

Muntendam, Antje

Muradás-Taylor, Becky

Mutta, Maarit

Nagano, Tomonori

Namboodiripad, Savithry

Occhino, Corrine

Ojea, Ana

Olsson, Eva

Orozco, Rafael

Perez-Cortes, Silvia

Pérez-Leroux, Ana T.

Pérez-Vidal, Carmen

Peyton, Joy Kreeft

Pladevall, Elisabet

Polinsky, Maria
Prichard, Caleb

Pulido, Manuel Jesús Cardoso

Purser, Benjamin

Putnam, Michael

Rato, Anabela

Regan, Brendan

Requena, Pablo E.

Riccelli, Adrian

Rivadeneira, Marcela

Roca, Francesc

Romano, Antonio

Rubio, Fernando

Schoonmaker-Gates, Elena

Schuhmann, Katharina

Schulte, Kim

Schwegler, Armin

Scontras, Gregory

SHANG, GUOWEN

Simonet, Miquel

Slabakova, Roumyana

Sleeman, Petra

Solon, Megan

Spinu, Laura

Stoian, Claudia

Sung, Park-Johnson

Tagliamonte, Sali A.

Terzi, Arhonto

Thomas, Erik

Thomas, Minnie

Torgersen, Eivind Nessa

Travis, Catherine

Tsaroucha, Efthymia

Van Goethem, Kristel

Vasilescu, Ioana-Gabriela

Verspoor, Marjolijn

Vosburg, Nora

Vraciu, Alexandra

Walden, Patrick R.

Wang, Yu-Fang

Weekes, Brendan

Wu, Ruilin

Wyngaerd, Emma Vanden

$\mathrm{Xu}$, Feng

Yang, Chunsheng

Yoon, Tae

Zahler, Sara

Zárate-Sández, Germán

Zhan, Fangqiong

Zhang, Lan

Zyzik, Eve 